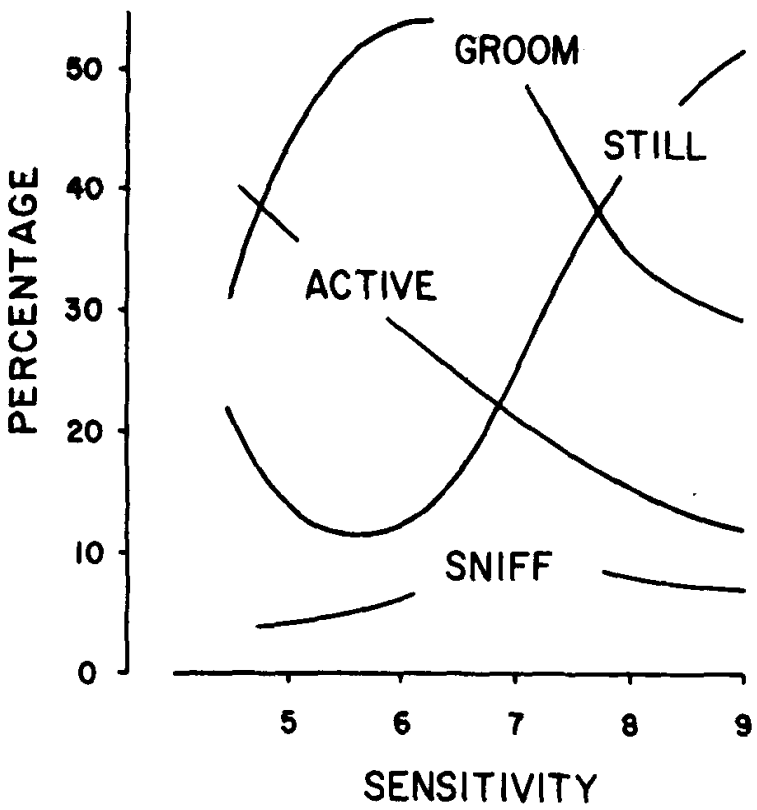

Fig. 2. The percentage of total daily counts to be expected from the ultrasonic device from the different behaviors of the rat as a function of the sensitivity setting.

only below sensitivities of approximately 5 , where most of rat's behaviors are below cutoff, that the most active forms of behavior can possibly make the dominant contribution to the total daily counts. At this point the ultrasonic device is responding to only a fraction of the rat's total behavior, and is probably counting essentially the same forms of behavior that the common photocell device would be counting. The chief difference would be that whereas the photocell detects a sample of instances of locomotion and rearing, the ultrasonic device would presumably detect all instances of these behaviors. Thus, whereas the photocell may generate a few hundred counts a day, the ultrasonic device would be generating up to 50,000 counts a day.
We do not know what sensitivities other Es have used with the ultrasonic device (the difficulty of specifying the sensitivity setting of it is one serious limitation), but Peacock and Hodge (1962) mention obtaining 100,000 counts a day, which amounts to about 1.2 counts per second. We may assume, then, that their sensitivity corresponded to a setting of approximately 6 in our situation, and that they were, therefore, counting mainly instances of grooming.

It could be argued that it does not matter too much what kinds of behavior the ultrasonic counts are based upon. As long as an experimental manipulation leads to more vigorous forms of behavior, an ultrasonic activity recorder will show an increase in the total number of counts. This is true enough as far as it goes, but it is now known that certain common types of experimental manipulations lead not so much to overall increases in activity but rather to changes in the pattern of activity (Bolles, 1965). Thus, for one example, water deprivation reduces grooming and increases wakeful lying still. Water deprivation might be expected, therefore, to decrease total daily counts at all but the highest sensitivity settings. As another example, we may suppose that eating, which occupies approximately $9 \%$ of the ad lib rat's time, will generate counts at a rate somewhere between sniffing and grooming, and that after food deprivation, such behavior must be replaced by a lot of quite vigorous behavior, or there would again be a net loss of counts at most sensitivity settings. Such anomalies can be expected, of course, with any activity measuring device that does not tell us precisely what $S$ is doing when it is being "active."

\section{REFERENCES}

BOLLES, R. C. Effects of deprivation conditions upon the rat's home cage behavior. Joumal of Comparative \& Phy siological Psy chology, 1965, 60, 244-248.

BOLLES, R. C. Theory of motivation. New York: Harper \& Row, 1967. PEACOCK, L. J., \& HODGE, M. An ultrasonic activity recording device. American Journal of Psychology, 1962, 75, 648-652.

NOTE

1. Supported by Research Grant GB-5694 from the National Science Foundation.

\title{
Liquid level detector with automatic refill and depletion indicator'
}

\section{DAVID KRAUSMAN, THE JOHNS HOPKINS UNIVERSITY SCHOOL OF MEDICINE, Baltimore, Maryland 21205}

An electromechanical device is described that provides the means for measuring the amount of water consumption from a storage device and for an automatic refill system. The instrument uses three sensing electrodes located in the storage bottle to drive a solid-state Darlington circuit, which, in conjunction with two liquid solenoid valves, controls the operation of the system.

In many behavioral experiments it is often necessary to measure the exact amount of water intake of animals over many hours, days, or even months of experimentation. Most manual methods of liquid refilling are inexact when the measurement of precise volumes are needed and require regular attention to insure that the water supply is never exhausted.

The solid-state detector described herein provides the $\mathrm{E}$ with a very accurate and precise technique for controlling automatically the refill of the water supply reservoir and recording the exact amount of water consumed over long time periods.

In operation, water is supplied from a graduated container by gravity feed. When the water level falls to a lower limit an open circuit occurs between two of the electrodes and a fill solenoid is activated. The outlet of the container is closed during the fill 


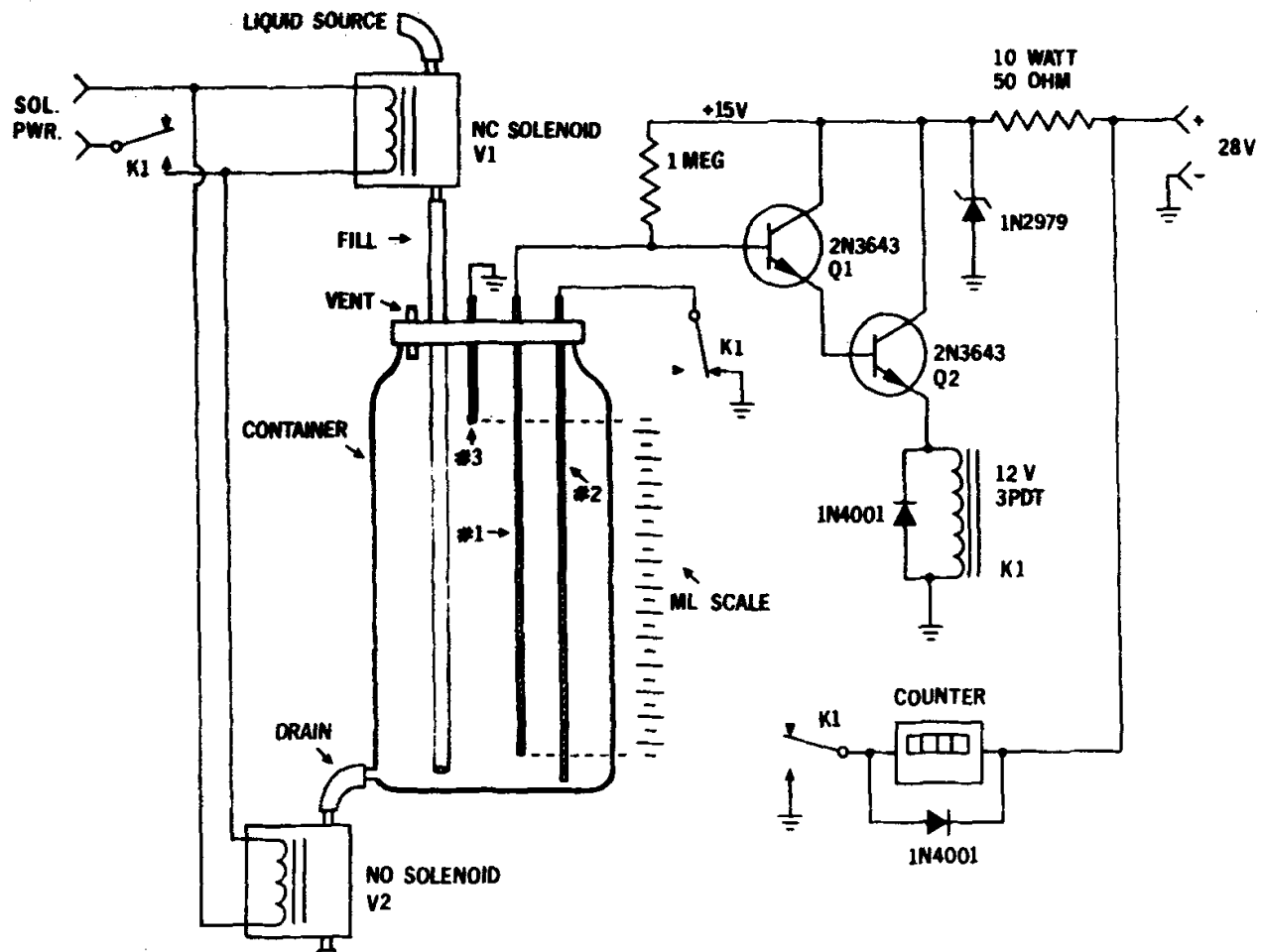

To LIQUD intake DeVICE

Fig. 1. Schematic diagram of the liquid-level detector.

cycle and the third electrode senses when the container is full. An electromagnetic counter tallies the number of emptyings and the total volume consumed is the sum of the total volumes emptied plus the scaled amount consumed at any time.

The supply reservoir (Fig. 1) should be made of transparent or semitransparent plastic, vinyl, or glass and should not be electrically conductive. The three electrodes are made of any stiff nonoxidizing wire and are fastened to a nonconductive lid for the container.

The vertical distance between the terminal end of Electrode 1 and Electrode 3 will determine the amount of water replenished in each filling cycle. When the water level breaks contact between the common Electrode 1 and Electrode 2, the refill cycle begins and will end as soon as the liquid level makes contact with Electrode 3. A calibrated milliliter scale attached to the container shows the volume of water between Electrodes 1 and 3 .

The operation of the circuit shown in Fig. 1 is as follows: A low-pressure water source is connected to the input of the normally closed solenoid valve, V1. The output of the solenoid, $V 1$, is connected to a fill tube that enters the container through the lid and extends almost to the bottom to avoid splashing during the filling cycle. The output port at the bottom of the container is connected to a normally open solenoid valve, V2. The fluid output of the V2 solenoid is connected to the animal's water intake device. Both Solenoids V1 and V2 are in parallel and are energized only during the filling cycle through the contacts of Relay K1. The combination of a normally closed V1 and a normally open V2 solenoid valve prevents the animal from consuming water during the filling cycle.

When the water level is somewhere between Electrodes 1 and 3 the solid-state Darlington amplifier consisting of Transistors Q1 and Q2 is in the "off" state due to the low resistance path between base (Electrode 1) and ground (Electrode 2) through the normally closed contacts of $\mathrm{K} 1$. This resistance is in the order of $10 \mathrm{~K}$ for tap water and, in conjunction with the l-megohm resistor, provides a 100:1 voltage divider at the base of $Q 1$, thereby holding the Darlington circuit and $\mathrm{K} 1$ in the "off" state.
As the water falls to a point where it breaks contact with Electrode 1, the Darlington amplifier is forward biased through the 1-megohm base resistor and switches the circuit to the "on" state energizing Control Relay K1.

When Control Relay $K 1$ is energized, two circuit functions are initiated: the two solenoid valves, $\mathrm{V}_{1}$ and $\mathrm{V} 2$, are actuated starting the filling cycle, and the circuit between Electrode 2 and ground is opened. The filling cycle will continue until the water level contacts Electrode 3, at which time the Darlington circuit will tum "off" causing Control Relay K1 to de energize, thus completing the filling cycle.

An impulse counter is powered through the contacts of Control Relay K1 and records the number of filling cycles. For example, if at the end of a 7-day experiment, the impulse counter reading was 5 and the volume between Electrodes 1 and 3 was $200 \mathrm{ml}$, the total water intake would be $5 \times 200 \mathrm{ml}$ plus the amount of water depleted after the last filling read from the milliliter scale.

A simple modification may be made to control daily water intake to a fixed volume. The impulse counter should be replaced with a predetermined type of impulse counter, the normally open output contacts of which should be connected between the base of Q1 and ground. Presetting the counter to the desired number of filling cycles will cause the counter when reaching zero to close the output contacts, thereby latching the Darlington circuit to the "off" state. No more water will be available to the animal until the $E$ resets the predetermined counter, which wil] automatically start a new filling cycle.

The solid-state Darlington detector circuit should work well with any liquid that has a resistance from 0 to $200 \mathrm{~K}$. If concurrent low-level physiologic recordings are made, the power supply for the circuit shown should be isolated to avoid artifact.

\section{NOTE}

1. This study was supported by PHS Grant HE 06945. Address requests for reprints to the author at: The Johns Hopkins University, Traylor Building, Room 617, 720 Rutland Avenue, Baltimore, Maryland 21205. 